\title{
Association between muscle trauma and heterotopic ossification in spinal cord injured patients: reflections on their causal relationship and the diagnostic value of ultrasonography
}

\author{
M Snoecx, M De Muynck, M Van Laere \\ Department of Physical Medicine and Rehabilitation, University Hospital, De Pintelaan 185, 9000 Ghent, \\ Belgium
}

\begin{abstract}
Paraplegic patients presenting with a subacute limitation of hip joint mobility were subjected to serial sonographic examinations. In four patients the initial sonographic study disclosed discontinuity with fluid collection in the psoas muscle, which was diagnostic of a traumatic muscle rupture. All four patients subsequently developed sonographic and radiographic evidence of heterotopic ossification. Our findings confirm that ultrasonography is an easy and inexpensive screening method for the early diagnosis of heterotopic ossification. The sonographic results obtained in these four paraplegic patients are indicative of a possible traumatic origin of heterotopic ossification around the hip.
\end{abstract}

Keywords: heterotopic ossification; muscle trauma; ultrasonography; risk factors; spinal cord injuries; early diagnosis

\section{Introduction}

Heterotopic ossification (HO) is a dreaded complication of traumatic spinal cord injury. Its incidence in a traumatic paraplegic population ranges between 16 and $53 \%,{ }^{1}$ dependent on the study design and the methods of detection used. HO always develops below the level of the spinal cord lesion.

Areas of predilection for ectopic bone formation are the large joints, predominantly the hips, elbows and knees. ${ }^{2}$ The clinical spectrum ranges from an incidental radiographic finding to severe limitation of the range of joint motion and even complete ankylosis. ${ }^{3}$

The etiology is still unknown. Both central and local mechanisms have been implicated. Central mechanisms could be genetic, hormonal or metabolic in nature. ${ }^{4-6}$ Possible local causes include microtraumatic lesions, immobilisation, infection, pressure ulcers and vasomotor disturbances. ${ }^{4-6}$

The most widely used method for the early identification of $\mathrm{HO}$ is three-phase radionuclide bone scanning. ${ }^{7}$ Major drawbacks of this examination are the expense of the equipment, the use of radioactive substances and the low specificity. Moreover, deep venous thrombosis (the most important differential diagnostic factor) cannot be ruled out, so further investigations are mandatory. ${ }^{?}$

Ultrasonography is considered to be an accurate tool for the examination of muscles and soft tissues. ${ }^{7}$ In recent years, it has gained more and more ground as the preferred initial imaging modality for patients with clinically suspected HO. ${ }^{7,8}$ The unique pathological evolution of the ossification can be monitored by sequential sonographic assessment.

In our department we have 6 years' experience with ultrasonography of the locomotor system. Paraplegic patients presenting with a subacute limitation of joint mobility below the level of injury are always subjected to a sonographic examination to exclude the onset of HO.

\section{Patients and methods}

Four male patients, ranging in age between 21 and 35 years, with complete traumatic paraplegia were included in the study. Two of them had complete T5 paraplegia; the other two had complete paraplegia at T8 and T9 respectively. The degree of lower extremity spasticity ranged from grade 0 to grade 3 on the Ashworth scale. Three of the four patients were on anticoagulant therapy to prevent the occurrence of deep venous thrombosis (Table 1 ).

All patients presented with the early clinical features of HO: subacute limitation of hip joint mobility, and localised swelling and soft tissue inflammation. In one patient the clinical symptoms were bilateral.

The onset was within 1-8 months of injury. On clinical suspicion of $\mathrm{HO}$ a sonographic investigation was performed, followed by three-phase radionuclide bone scanning and a routine radiographic examination.

The sonographic examination, using a $7.5 \mathrm{mHz}$ linear transducer probe (Siemens sonoline SL1 Erlangen, Germany), was always done by the same examiner in the Department of Physical Medicine and Rehabilitation within $48 \mathrm{~h}$ of clinical presentation. Serial follow-up examinations were done at weekly intervals. A repeat three-phase radionuclide bone scan was obtained 1-2 months after the initial one to assess bone maturation. The hip joint was examined sonographic- 
Table 1 Details of four patients with subacute limitation of hip joint mobility

\begin{tabular}{|c|c|c|c|c|c|c|c|c|}
\hline & $\begin{array}{c}\text { Level of } \\
\text { injury }\end{array}$ & Age & Sex & $\begin{array}{c}\text { Months } \\
\text { post-injury }\end{array}$ & $\begin{array}{c}\text { Location } \\
\mathrm{HO}\end{array}$ & $\begin{array}{l}\text { Spasticity } \\
\text { Ashworth }\end{array}$ & $\begin{array}{c}\text { Alk. } \\
\text { Phosph. }\end{array}$ & Antico \\
\hline 1 & $\mathrm{~T} 8$ & 33 & $\mathbf{M}$ & 1 & $\mathrm{R}$ hip & 1 & No & + \\
\hline 2 & $\mathrm{~T} 5$ & 21 & M & 2 & R hip & 0 & $\uparrow$ & + \\
\hline 3 & $\mathrm{~T} 5$ & 35 & M & 8 & $\mathrm{R}+\mathrm{L}$ hip & 3 & $\uparrow$ & - \\
\hline 4 & T9 & 33 & $\mathbf{M}$ & $21 / 2$ & $\mathrm{R}+\mathrm{L}$ hip & 2 & No & + \\
\hline
\end{tabular}

ally following a standardised protocol. A right/left comparison was always made. The linear transducer probe was placed in a ventral-vertical and a lateralvertical position. The underlying bony structures were identified for orientation purposes. Proximally, the acetabular rim and more distally the femoral head and neck were noted as an echogenic white band with an underlying sonolucent shadow. The superjacent muscle tissue appeared as a homogeneous lamellar echogenic structure (Figure 1).

The sonographic findings were considered abnormal if a chaotic inhomogeneous sonographic pattern was seen instead of the normal homogeneous lamellar structure of muscle tissue.

\section{Results}

The results are summarised in Table 2. All four paraplegic patients presented with the early features of HO. The site of involvement was always the hip. One patient had bilateral symptoms.

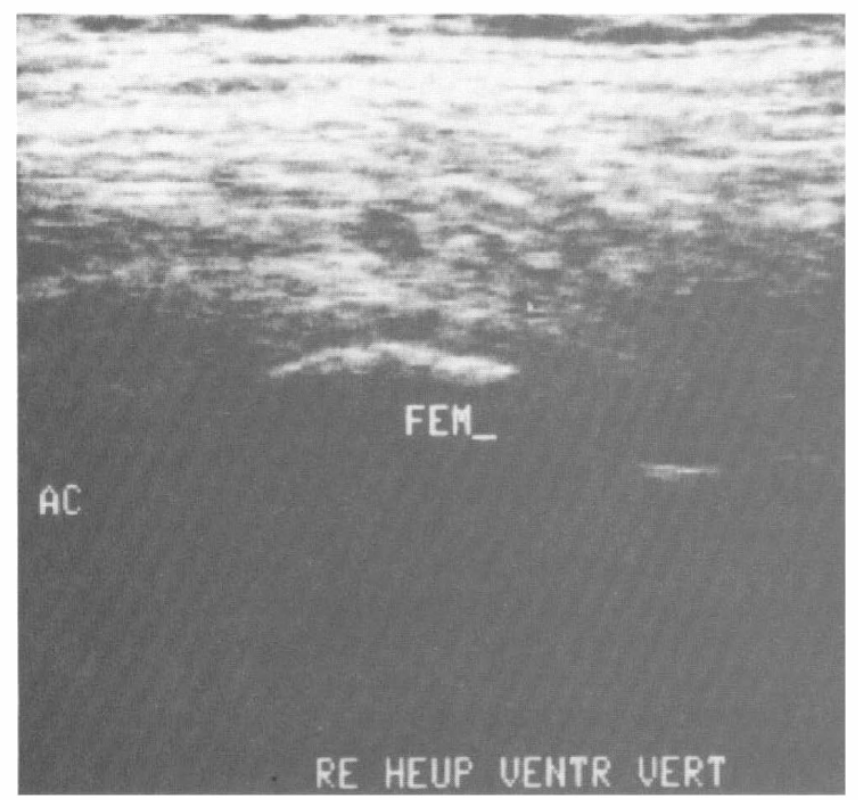

Figure 1 Ultrasound appearances of a normal hip: the underlying bony structures are identified for orientation purposes. Proximally, the acetabular rim and more distally the femoral head and neck are noted as an echogenic white band with an underlying sonolucent shadow. The superjacent muscle tissue of the psoas appears as a homogenous lamellar echogenic structure
The initial examination disclosed abnormal findings in six hip joints. One patient had bilateral sonographic abnormalities, although his complaints were confined to the right hip. In five of the six hips marked swelling of the psoas muscle with intramuscularly a large hypoechoic to echolucent zone was initially observed. This sonographic appearance is suggestive of a partial muscle tear with massive haemorrhage (Figure 2). In this hypoechoic to echolucent zone distinct calcifications were noted in two hips, presenting as echogenic bands with acoustic shadowing in the depth. In two other hips with a suspected partial muscle tear one or more additional, slightly more echogenic bands without shadowing were seen, which appeared to be suggestive of the onset of calcifications (Figure 3). The fifth hip only presented a markedly swollen psoas muscle and a considerable sonolucent zone. The sixth hip, in which features of a torn muscle were absent, showed sonographic evidence of an additional subcutaneous mass of echogenic foci with a surrounding oedematous zone. Further sonographic differentiation proved to be impossible. The differential diagnosis of subcutaneous haematoma, fat necrosis or an early abscess was advanced.

Within 14 days two patients underwent a follow-up sonographic examination. The hypoechoic to echolucent zone was found to be considerably reduced to smaller well-delineated echolucent zones with marked discontinuity of the muscle tissue. Moreover, the echogenic bands were more pronounced and larger in number, producing acoustic shadowing in the depth, which was indicative of increasing calcification (Figure 4). These echogenic bands encompassed a hypoechoic zone.

On a subsequent sonographic examination of the first patient the fluid collections could no longer be visualised, but several distinct calcifications were seen, which became increasingly reflective and confluent. Finally, these calcifications reflected as a homogeneously echogenic band, behaving like normal corticated bone (Figure 5).

In all of the hips with initial sonographic abnormalities, HO eventually developed and was confirmed both radiographically and by three-phase radionuclide imaging.

\section{Discussion}

Ultrasonography is generally considered to be a highly accurate method for the evaluation of muscles and soft 


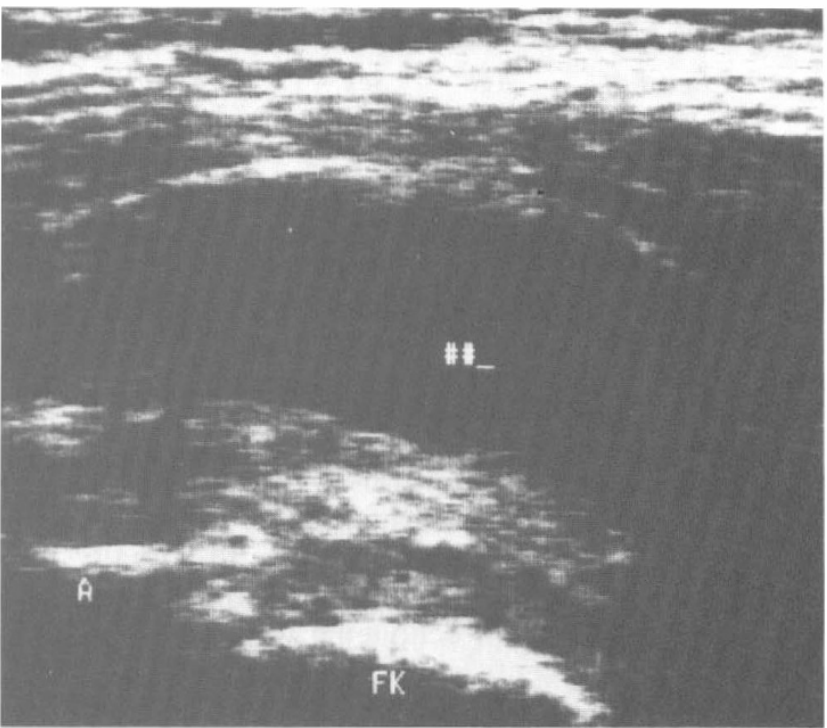

Figure 2 First abnormal sonographic findings: the superjacent muscle tissue appears no longer as a homogenous lamellar echogenic structure; instead we observe intramuscularly into the psoas muscle a large hypoechoic to echolucent zone $\left({ }^{* *}\right)$. This sonographic appearance is suggestive of a partial muscle tear with massive haemorrhage

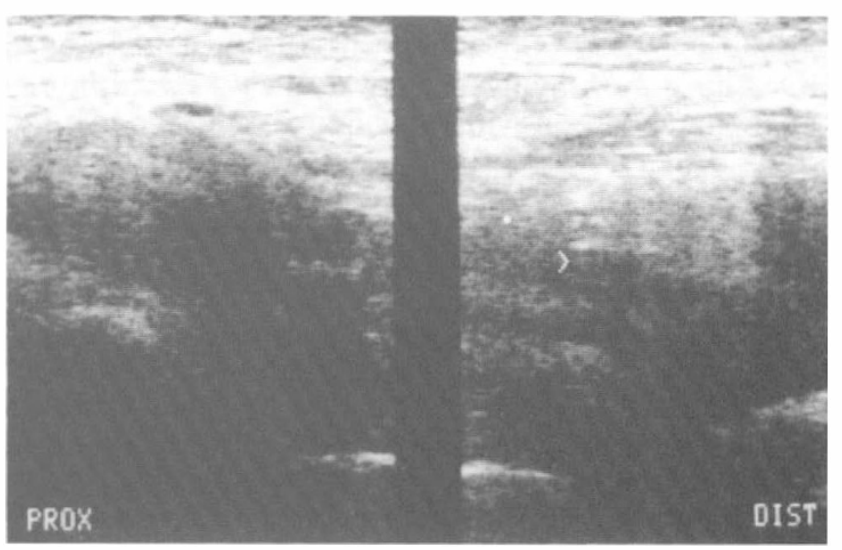

Figure 3 First abnormal sonographic findings: more distally, slightly more echogenic bands without shadowing are seen $(>)$. These echogenic bands are suggestive of early calcification

tissues. It is an inexpensive, non-invasive, transportable, highly reproducible imaging modality with a high specificity and sensitivity. Its only drawback is that it depends strongly on experience.

Ultrasonography is being more and more adopted as the primary study for the documentation of clinically suspected $\mathrm{HO}{ }^{7}$ Not only can the typical abnormalities be uncovered prior to their demonstration on routine radiographs, but serial sonographic monitoring of disease activity also allows us to follow the evolution to complete ossification. ${ }^{7}$

Three-phase radionuclide scanning is also positive in the early stages, but the investigation is non-specific, expensive and necessitates patient transportation. 


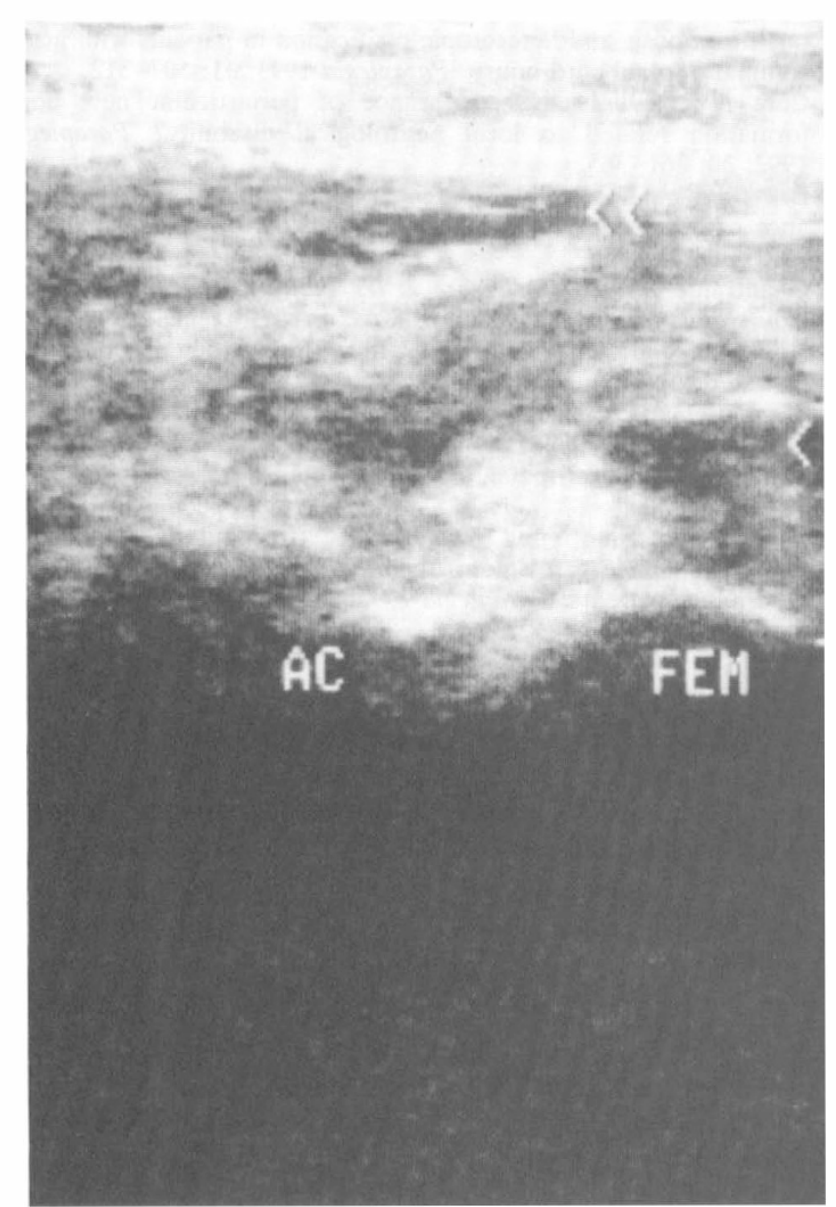

Figure 4 Follow-up sonographic examination: after 14 days the large hypoechoic to echolucent zone is considerably reduced. Smaller well delineated echolucent zones with marked discontinuity of the muscle tissue are observed. The echogenic bands are more pronounced and larger in number

Scintiscanning is probably mainly valuable in assessing the maturation of $\mathrm{HO}$, when surgical removal is comtemplated. ${ }^{9}$

During our 6 years' experience with ultrasonography of the locomotor system 39 patients were referred to us with clinical suspicion of $\mathrm{HO}$ of the hip. In four patients the initial sonographic findings were consistent with a traumatic muscle tear. Only afterwards did we observe what was described by Ackermann and Johnson as the zone phenomenon: a central amorphous hypoechoic zone surrounded by a non-uniform, more echogenic zone. The latter zone was initially not uniformly echogenic, but contained foci of echogenic islands which rapidly became confluent. As they became more and more confluent they also became increasingly reflective due to increasing mineralisation. With further mineralisation the ultrasound beam was totally reflected because the lesion behaved like corticated bone; at this stage the radiographs became positive, ie after an average of 4-6 weeks.

In our four paraplegic patients the initial sonographic

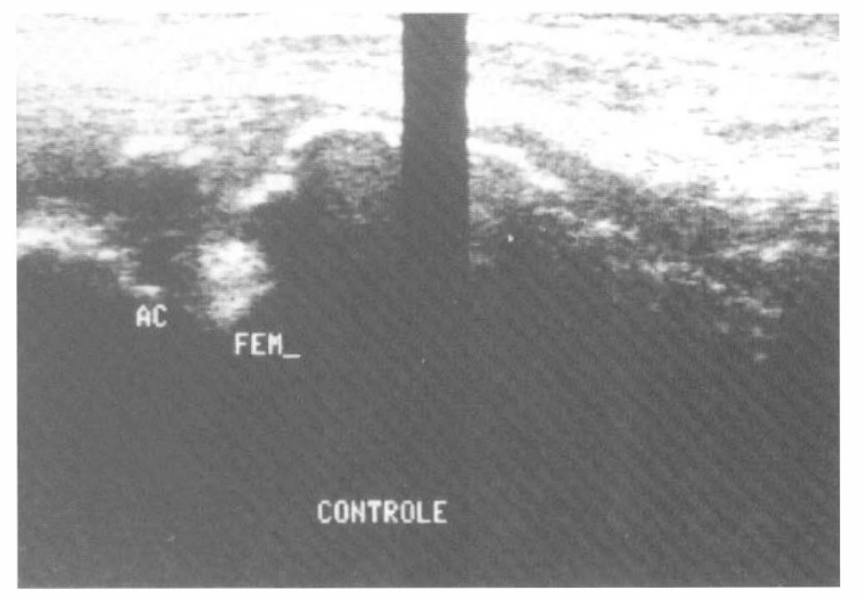

Figure 5 Follow-up sonographic examination after 12 months: the fluid collections have disappeared. Several calcifications are noticed. These large calcifications reflect as a homogenously echogenic band behaving like normal cortical bone

examination did not always demonstrate the zone phenomenon. It was not until a few days to 1 week that the sonographic pattern characteristic of early $\mathrm{HO}$ became apparent. The sonographic appearance in our four patients is important because it may indicate a possible causal relationship between microtraumatic lesions (eg a traumatic muscle tear) and the occurrence of $\mathrm{HO}$.

Recent literature data support this hypothesis. In their histological study of the zone phenomenon, Cassar-Pullincino et al demonstrated the presence of muscle necrosis, haemorrhage and undifferentiated cellular proliferation in the central hypoechoic zone. ${ }^{8}$ The intermediate, more echogenic zone contained immature bone and osteoblasts. As the lesion matured, immature bone with a trabecular configuration could be seen in the outer zone. After some 2 weeks osteoid formations were laid down in a zonal and centripetal pattern, finally yielding mature bone with marrow cavity.

A histological study, conducted by Bodley, Jamous and Short with needle biopsy of the zone phenomenon, also showed small osteoid formations and early bone formation, without calcification and with haemosiderin-loaded macrophages, as evidenced by Perl's staining. ${ }^{7}$ These haemosiderin-loaded macrophages might indicate resorption of a haematoma. Both Bodley and Cassar-Pullicino concluded from their histological studies that microtraumatic lesions might be a causative factor in the occurrence of $\mathrm{HO}$.

In retrospect, no such factor could be demonstrated in our four paraplegic patients, but all were in the stage of intensive rehabilitation and transfer activities.

No relationship could be established with the degree of spasticity, although all four patients presented with complete thoracic lesions; three patients were still on anticoagulant therapy. 


\section{Conclusion}

The early sonographic appearance and findings in seven paraplegic patients support the hypothesis that HO might be traumatic in origin, and stress the diagnostic value of ultrasonography in the early identification of clinically suspected $\mathrm{HO}$.

\section{References}

1 Colachis SC, Clinchot DM, Venesy D. Neurovascular complications of heterotopic ossification following spinal cord injury. Paraplegia 1993; 31: 51-57.

2 Wittenberg RH, Peschke U, Bötel U. Heterotopic ossification after spinal cord injury: epidemiology and risk factors. $J$ Bone Joint Surg Br 1992; 74: 215-218.

3 Colachis SC, Clinchot DM. The association between deep ven-

ous thrombosis and heterotopic ossification in patients with acute traumatic spinal cord injury. Paraplegia 1993; 31: 507-512.

4 Catz A et al. Is the appearance of periarticular new bone formation related to local neurological disability? Paraplegia 1992; 30: 361-365.

5 Bravo-Payno $\mathrm{P}$ et al. Incidence and risk factors in the appearance of heterotopic ossification in spinal cord injury. Paraplegia 1992; 30: 740-745.

6 Chantraine A. L'Osteoporose et les Para-osteo-arthropathies au Cours de la Paraplégie. Editions Arsia SA: Bruxelles, 1979.

7 Bodley R, Jamous A, Short D. Ultrasound in the early diagnosis of heterotopic ossification in patients with spinal injuries. Paraplegia 1993; 31: 500-506.

8 Cassar-Pullicino VN et al. Sonographic diagnosis of heterotopic bone formation in spinal injury patients. Paraplegia 1993; 31: $40-50$.

9 Sun W Kim, Sing Yung Wu, Kim RC. Computerized quantitative radionuclide assessment of heterotopic ossification in spinal cord injury patients. Paraplegia 1992; 30: 803-807. 